\title{
DESENVOLVIMENTO DE MODELO CONCEITUAL DE SISTEMAS DE CUSTOS - UM ENFOQUE INSTITUCIONAL
}

\author{
DEVELOPMENT OF CONCEPTUAL MODEL SYSTEM COST - AN \\ INSTITUTIONAL APPROACH
}

\begin{abstract}
WELINGTON ROCHA
Doutor em Controladoria e Contabilidade pela Universidade de São Paulo Professor da Universidade de São Paulo, Faculdade de Economia Administração e Professor da Universidade de São Paulo, Faculdade de Economia Administração e
Contabilidade, Departamento de Contabilidade Atuária - São Paulo/SP, Brasil. E-mail: cmslab@usp.br
\end{abstract}

\section{Resumo}

O estudo demonstra que o modelo de institucionalização de Burns e Scapens (2000), especialmente no que se refere ao processo de codificação de rotinas e regras, pode ser aplicado para analisar a implementação de um novo sistema de contabilidade gerencial em um grande banco brasileiro. Como aquele modelo é relativamente recente, poucos estudos utilizaram sua estrutura conceitual em pesquisas empíricas. Alguns (Soin et al., 2002; Spraakman, 2006; Guerreiro et al., 2006) adotaram o que pode ser denominado perspectiva institucional para estudar vários aspectos da Contabilidade Gerencial, mas o presente artigo é o primeiro a focar especificamente a modelagem da fase inicial do processo de mudanca no setor bancário a partir de uma perspectiva da Teoria Institucional. Utilizou-se o método de estudo de caso para avaliar os estágios embrionários do processo de implementação. Os dados foram coletados por meio de entrevistas e análise documental a respeito dos seguintes aspectos: (i) os antecedentes do processo de mudança; (ii) as características operacionais e organizacionais do banco e (iii) o processo de modelagem usado para conceber o sistema. As principais conclusões foram que: (a) a visão institucional é tão importante quanto a dimensão técnica-racional na fase de concepção do novo modelo de custeio; (b) o processo de codificação deve especificar regras e rotinas que sejam coerentes com as crenças institucionais e com as práticas dos membros do staff e (c) a probabilidade de sucesso é significativamente incrementada quando os novos sistemas são modelados em termos do modelo adaptado de Burns e Scapens (2000). Finalmente, o estudo apresenta uma nova visão a respeito da implementação de sistemas de custeio em geral, com mais foco na dimensão institucional do que na dimensão racional-normativa.

Palavras chave: Teoria Institucional, sistemas de custeio, Contabilidade Gerencial.

\section{Abstract}

The present study demonstrates that the institutionalisation model of Burns and Scapens (2000), especially the 'encoding' process of rules and routines, can be applied to analyse the successful implementation of a comprehensive new management accounting system in a large Brazilian financial institution. As that model is relatively recent, few studies have utilised this theoretical framework in empirical research. Some (Soin et al., 2002; Spraakman, 2006; Guerreiro et al., 2006) have adopted what might be called an 'institutional perspective' in studying various aspects of management accounting, but the present study would appear to be the first to have specifically focused on the initial modelling phase of a change process in the banking sector from the perspective of institutional theory. It was applied the case study method to assess the formative stages of the implementation process. Data were collected by means of interviews and documentary analysis on the following issues: (i) the antecedents of the change process; (ii) the organisational and operational characteristics of the bank; and (iii) the modelling process used to conceive the system. The main conclusions are that: (a) the institutional dimension was regarded by the case firm as being as important as the technical-rational dimension in the conception phase of the new costing model; (b) the encoding process should specify rules and routines that are coherent with the institutional beliefs and practices of staff members and (c) the likelihood of success will be significantly enhanced if firms model their new systems in terms of the adapted institutional model of Burns and Scapens (2000). Finally, the study provides new insights into costing systems implementation in general, with a closer focus on the institutional dimension than on the rational-normative dimension.

Keywords: institutional theory, cost system, management accounting, Brazil. 


\section{INTRODUÇÃO}

Observa-se atualmente um acúmulo de pesquisas sobre mudanças organizacionais e principalmente sobre mudanças em contabilidade gerencial. No ambiente de negócios, pesquisadores e consultores têm adotado uma postura normativa, argumentando que as empresas devem utilizar novos sistemas de contabilidade gerencial identificados com acrônimos, tais como $A B C, A B M$, EVA, TOC, dentre outros. Muitas empresas têm implementado esses novos artefatos de contabilidade gerencial com diferentes graus de sucesso (COHEN; PAQUETTE, 1991; BRIGTH et al., 1992; DRURY; TAYLES, 1995; KASURINEN, 2002).

No âmbito acadêmico, diversos pesquisadores têm desenvolvido estudos para explicar por que e como ocorrem as mudanças na contabilidade gerencial (SCAPENS, 1994; LIBBY; WATERHOUSE, 1996; GRANLUND; LUKKA, 1998; EDWARDS; EMMANUEL, 1990; EMORE; NESS, 1991; GREEN; AMENKHIENAN, 1992; BURNS; SCAPENS, 2000; GRANLUND, 2001; SOIN, SEAL; CULLEN, 2002; GUERREIRO; PEREIRA; FREZATTI, 2006; SPRAAKMAN, 2006) e recomendam diversos arcabouços conceituais, vindos de fora da área contábil, para explicar essas mudanças (COVALESKI, DIRSMITH, SAMUEL, 1996; QUATTRONE; HOPPER, 2001; GUERREIRO; CASADO; BIO, 2004; SCAPENS, 2006).

Os estudos desenvolvidos em ambientes de serviços e, principalmente, no ambiente de serviços bancários, tais como o de Cobb, Helliar e Innes (1995) e o de Helliar, Cobb e Innes (2002), são em menor volume, muito embora se observe que o setor de serviços vem desempenhando um papel relevante na economia dos países desenvolvidos (BRIGNALL, 1997) e em desenvolvimento. O presente estudo investiga a adoção do sistema de custos em ambiente bancário, utilizando como arcabouço conceitual a Velha Economia Institucional (Old Institutional Economics - OIE). Sob esse enfoque, o sucesso da introdução de novos conceitos ocorre quando eles são institucionalizados, ou seja, quando são convertidos em novos valores, hábitos e rotinas dentro da organização (OLIVER, 1997).

Uma estrutura conceitual para análise do processo de institucionalização das mudanças na contabilidade gerencial, segundo a abordagem da Velha Economia Institucional, foi proposta por Burns e Scapens (2000) a partir do estudo de Barley e Tolbert (1997). Esse processo apresenta quatro etapas fundamentais: codificação, incorporação, reprodução e institucionalização. O modelo demonstra a ligação entre o campo institucional e o campo da ação através da dinâmica dessas quatro fases.

Este trabalho tem dois objetivos fundamentais. 0 primeiro é apresentar o estado da arte das pesquisas e estudos sobre a teoria institucional no contexto da Velha Economia Institucional, a partir de uma profunda revisão de literatura. A apresentação desse referencial teórico se caracteriza como um primeiro objetivo importante na medida em que, tradicionalmente, a concepção e a implantação de sistemas de contabilidade gerencial têm sido fortemente influenciadas pela abordagem normativa, fortemente prescritiva. Ainda é incipiente, principalmente no Brasil, o emprego de teoria institucional nos estudos da contabilidade gerencial.

O segundo objetivo é apresentar os resultados de pesquisa empírica realizada 
em instituição financeira atuando no Brasil, com base na metodologia de estudo de caso, investigando o processo de modelagem do seu sistema de custos a partir da estrutura conceitual proposta por Burns e Scapens (2000).

O foco do estudo é o entendimento de como ocorreu o processo de codificação das regras e rotinas do sistema de custos no ambiente do banco estudado. Este banco foi escolhido como objeto de estudo pelas seguintes razões: (i) sua importância no cenário econômico brasileiro, (ii) a possibilidade de obtenção de dados por parte dos pesquisadores e (iii) o banco passou por uma experiência importante, com a introdução do novo sistema de custeio na corporação.

Tendo em vista que o modelo de Burns e Scapens (2000) é relativamente recente, são poucos os estudos que têm utilizado esse arcabouço teórico em estudos empíricos, destacando-se os trabalhos de Soin, Seal e Cullen (2002), Spraakman (2006) e Guerreiro, Pereira e Frezatti (2006). A contribuição deste estudo se dá à medida que, na revisão de literatura, não foi observado nenhum estudo focando especificamente a fase da modelagem de sistema de contabilidade sob o prisma da teoria institucional.

Além desta introdução, o trabalho apresenta o referencial teórico empregado, abordando os aspectos fundamentais da Velha Economia Institucional, a apresentação do caso estudado, a discussão do caso à luz do referencial teórico e, por último, são apresentadas as conclusões.

\section{REFERENCIAL TEÓRICO}

\subsection{VELHA ECONOMIA INSTITUCIONAL (OLD INSTITUCIONAL ECONOMICS)}

Burns e Scapens (2000) afirmam que o interesse pela teoria institucional em ciências sociais vem aumentando e pelo menos dois enfoques dessa teoria podem ser observados na literatura contábil: (i) Nova Sociologia Institucional (New Institutional Sociology - NIS) e (ii) Velha Economia Institucional (Old Institutional Economics - OIE). De acordo com Burns (2000), a teoria institucional que fundamenta estudos analíticos de mudanças nas rotinas dos sistemas de contabilidade gerencial é a Velha Economia Institucional (OIE) - a qual se constitui em um corpo teórico muito heterogêneo.

A abordagem institucional rejeita os postulados da teoria neoclássica como principal direcionador para o entendimento das práticas da contabilidade gerencial. Considerando-se a teoria institucional segundo a óptica da OIE, a instituição é o principal objeto de análise e não mais o comportamento racional e maximizador dos indivíduos tomadores de decisões, conforme aceito pela teoria neoclássica (SCAPENS, 1994).

Não existe uma definição simples e amplamente aceita de instituição. Burns e Scapens (2000, p. 8) definem instituição a partir da versão de Barley e Tolbert (1997), ou seja, como “[...] pressuposições compartilhadas e aceitas de forma inquestionável, as quais identificam categorias de atores humanos e suas atividades 
e relacionamentos apropriados." Scapens (1994, p. 305) menciona que, no âmbito da OIE, a primeira definição de instituição foi dada por Veblen em 1919: “[...] um determinado modo de pensar comum para um conjunto de pessoas." Segundo Burns (2000), a ideia de instituição mais aplicada na OIE foi dada por Walton Hamilton, em 1932, que considera instituição como uma forma de pensar ou agir que prevalece e permanece, e está inserida nos hábitos de um grupo ou nos costumes de um povo. Essa definição traz à tona o caráter social e cultural do conceito de instituição e enfatiza a importância do comportamento habitual.

As ideias de hábito e de instituição estão conectadas pelo conceito de rotina. A caracterização de hábitos envolve uma predisposição ou tendência para se engajar em formas de ação anteriormente adotadas ou adquiridas. É importante observar que a existência de hábitos não exclui a ideia de intencionalidade do comportamento individual e não significa que hábitos não possam ser modificados. Embora hábitos estejam na esfera pessoal, rotinas podem envolver um grupo de pessoas (OLIVER, 1997) e, assim, tornar-se o principal componente das instituições. Em outras palavras, rotinas são hábitos formalizados e institucionalizados que incorporam comportamentos orientados por regras, fortalecendo-se pelo processo de repetição de ações para o atendimento das regras. Rotinas são formas de pensar e de agir, habitualmente adotadas por um grupo de indivíduos de forma inquestionável.

As regras e rotinas proporcionam uma memória da organização e se constituem na base para a evolução do comportamento organizacional. Segundo Scapens (1994), elas são equivalentes organizacionais dos genes no processo biológico e, nesse sentido, evolução não é a criação do comportamento ótimo, mas meramente a reprodução e possível adaptação de comportamentos através do tempo. Oliver (1997) enfatiza que, sob a perspectiva institucional, as empresas operam dentro de uma estrutura social de normas, valores e pressuposições assumidas (taken-for-granted) a respeito do que constitui o comportamento econômico apropriado ou aceitável. A visão institucional sugere que os motivos do comportamento humano se estendem para além da otimização econômica, à justificação e obrigação social. Oliver (1997) menciona que atividades institucionalizadas são aquelas ações que tendem a ser duradouras, socialmente aceitas, resistentes à mudança e não diretamente dependentes de recompensas ou do monitoramento da sua permanência.

No âmbito da contabilidade gerencial, Scapens (1994) enfatiza a ideia de institucionalização observando que a contabilidade gerencial pode, através do tempo, constituir-se em uma estrutura que reflete a maneira de pensar e agir de uma organização - a qual é aceita de forma inquestionável, sendo dissociada das suas circunstâncias históricas específicas. No trabalho de Guerreiro, Pereira e Frezatti (2006) é apresentado um conjunto de características específicas de uma instituição: (i) o caráter coletivo, isto é, as instituições são estruturadas por meio de rotinas definidas a partir de formas de pensar e de hábitos formalizados aceitos pelas pessoas de um determinado grupo social, (ii) o caráter de dar sentido ao comportamento organizacional e, nessa direção, as instituições permitem aos indivíduos e aos grupos dentro da organização dar sentido às suas atividades diárias, (iii) o caráter de definir padrões de comportamento esperados de um grupo social específico, (iv) o caráter normativo das instituições, geralmente refletindo regras estabelecidas na organização, muito embora isso nem sempre ocorra, porque algumas regras podem não ser aceitas 
pelo grupo social, (v) o caráter de não questionamento; nesse sentido os membros do grupo aceitam as instituições quase automaticamente, sem discussão, (vi) o caráter da repetição das instituições, implicando em hábitos, rotinas e, portanto, em repetição de ações, (vii) o caráter de estabilidade, sendo a duração de uma instituição muito variável; algumas instituições têm uma vida curta e outras, uma duração maior; no entanto, todas as instituiçõos duram determinado período de tempo, enquanto existe estabilidade, (viii) o caráter de orientar ações. Quanto mais amplamente e mais profundamente uma instituição é aceita, maior é a probabilidade de que possa exercer influência, orientando ações e resistindo a mudanças.

\subsection{O ENFOQUE INSTITUCIONAL (OIE) NA CONTABILIDADE GERENCIAL}

Burns e Scapens (2000) utilizam os conceitos de hábitos, rotinas e instituições para sugerir que as práticas contábeis podem se tornar rotineiras e, através do tempo, passar a fazer parte do conjunto das pressuposições e crenças inquestionáveis da organização (taken-for-granted). Essas pressuposições e crenças são profundamente instaladas na cultura do grupo social e aceitas de maneira automática, de forma que as pessoas nem cogitam indagar sobre elas. Práticas contábeis e rotinas emergentes podem ser caracterizadas como institucionalizadas quando se tornam amplamente aceitas na organização e são vistas como formas inquestionáveis de controle gerencial. Dessa forma, a contabilidade gerencial, como instituição, corresponde a um conjunto de rotinas institucionalizadas e aceitas na organização e tanto impacta outras instituições no âmbito da organização como é moldada por elas.

De forma geral, os sistemas de contabilidade gerencial podem ser vistos como uma importante rotina no âmbito das organizações ou um conjunto estruturado de rotinas, que estabelecem as diretrizes para as ações (alocação de recursos, decisões operacionais, decisões de preços etc.) e fornecem os meios para representar o desempenho dos diversos atores. Nesse contexto, a contabilidade gerencial, mais do que adotar os preceitos da teoria convencional e se materializar como geradora de informações para tomada de decisões racionais voltadas à maximização do lucro, estrutura uma base institucional para a tomada de decisões e para a formação de crenças e expectativas. A questão é o entendimento da maneira pela qual as práticas de contabilidade gerencial proporcionam coerência social, bem como significado ao comportamento organizacional, permitindo aos indivíduos e aos grupos da organização dar significado às suas atividades do dia-a-dia. Quando as práticas contábeis se tornam rotinas institucionalizadas, sua função no processo de gestão organizacional e na tomada de decisões é aceita pelos membros da organização. A contabilidade, dessa forma, torna-se um mecanismo que dá sentido às atividades empresariais e que é utilizado como mediador de potenciais conflitos dentro da organização.

Os estudos do enfoque institucional (OIE) na contabilidade gerencial tiveram início com o trabalho de Scapens e Roberts (1993) que explora aspectos antecedentes e consequentes da resistência encontrada pela equipe de desenvolvimento de projeto na introdução de um novo sistema de contabilidade gerencial. 0 trabalho de Scapens (1994) apresenta forte dose de pioneirismo à medida que propõe, de forma assertiva, o enfoque da Velha Economia Institucional e contesta o paradigma da teoria econômica neoclássica como orientação para o desenvolvimento de 
sistemas de contabilidade gerencial. Naquele trabalho, os argumentos, conceitos e elementos da teoria institucional como abordagem adequada para a compreensão da contabilidade gerencial são apresentados e discutidos. O estudo de Burns (1997) reforça a abordagem institucional e agrega novas reflexões, apresentando o estudo de caso (Keano) sobre institucionalização de rotinas contábeis. Os trabalhos de Scapens (1994) e Burns (1997) estabelecem os fundamentos para o estudo desenvolvido por Burns e Scapens (2000), no qual é apresentada uma estrutura conceitual para a análise do processo de institucionalização em contabilidade gerencial. Essa estrutura é analisada de forma detalhada no tópico 2.3 deste trabalho. Naquele mesmo ano, Burns (2000) apresenta um estudo sobre mudanças contábeis utilizando o enfoque institucional e incorporando o aspecto de poder.

A estrutura conceitual do processo de institucionalização desenvolvida por Burns e Scapens (2000) tem sido utilizada como referência para estudos de casos. Nesse contexto destaca-se a pesquisa de Soin, Seal e Cullen (2002). Esses autores utilizaram o modelo de Burns e Scapens (2000), em sua pesquisa, fundamentada em estudo de caso, para interpretar o papel da contabilidade gerencial na mudança organizacional em uma empresa do Reino Unido. O banco implantou um sistema de custeio baseado em atividades em um departamento de serviços de retaguarda, e o estudo é focado na análise das dicotomias de mudanças: formal versus informal, revolucionária versus evolucionária e regressiva versus progressiva.

O trabalho de Siti-Nabiha e Scapens (2005) enfoca a dualidade entre estabilidade e mudança na implementação de um novo sistema de gerenciamento baseado em valor em empresa de processamento de gás, utilizando a abordagem institucional. 0 trabalho de Guerreiro, Pereira e Frezatti (2006) utiliza o modelo de Burns e Scapens (2000) para analisar o processo de institucionalização da contabilidade gerencial no Banco do Brasil depois de sete anos de sua implantação. Spraakman (2006) utiliza a estrutura conceitual de Burns e Scapens (2000) para examinar os diferentes sistemas de contabilidade gerencial utilizados ao longo da existência da empresa Hudson's Bay Company. Lukka (2006), utilizando a abordagem de Burns e Scapens (2000), desenvolveu um estudo em que verifica a coexistência de mudança e estabilidade da contabilidade gerencial da empresa pesquisada.

\subsection{O MODELO DE INSTITUCIONALIZAÇÃO DE BURNS E SCAPENS}

Barley e Tolbert (1997) apresentam um modelo geral de institucionalização através da integração de duas bases teóricas: teoria institucional e teoria da estruturação. Os trabalhos de Giddens (1984) parecem ter sido importantes fontes de inspiração para a concepção do modelo de Barley e Tolbert (1997), no qual os conceitos de campo institucional e campo da ação são enfatizados. Barley e Tolbert (1997), de certa forma, transformaram o modelo estático de Giddens em um modelo dinâmico de estruturação social em que as ideias de instituição e de ação interagem em uma dimensão temporal, modificando os papéis dos atores, por meio dos processos de codificação, incorporação, replicação ou revisão, exteriorização e objetivação.

O trabalho de Burns e Scapens (2000) modifica o modelo inicial de Barley e Tolbert (1997), incorporando conceitos fundamentais da teoria institucional na forma 
da Velha Economia Institucional - hábitos, rotinas e regras - na busca da conceituação de mudança em contabilidade gerencial. Nesse contexto, a noção de papéis, que estava presente no modelo de Barley e Tolbert (1997), dá lugar aos conceitos de rotinas e regras no modelo de Burns e Scapens (2000), conforme apresentado na Figura 1, a seguir. Este modelo apresenta quatro processos fundamentais: codificação, incorporação, reprodução e institucionalização.

$$
\begin{array}{r}
\text { Legenda: } \\
a=\text { codificação } \\
b=\text { incorporação } \\
c=\text { reprodução } \\
\text { d = institucionalização }
\end{array}
$$

\section{Campo Institucional}

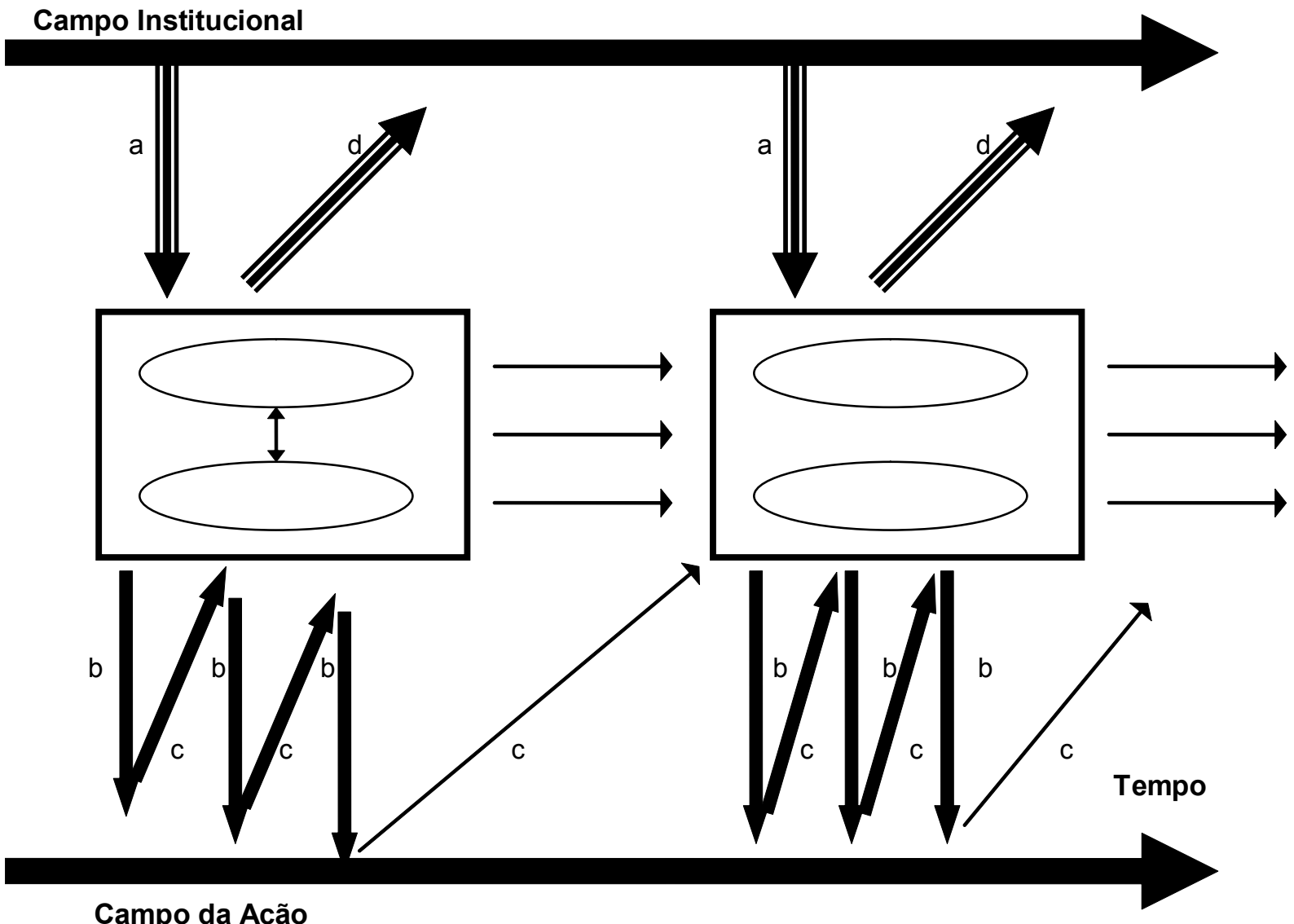

Figura 1: 0 processo de institucionalização

Fonte: Burns e Scapens (2000, p. 9)

A dinâmica do modelo demonstra a ligação entre o campo institucional e o campo da ação. Num primeiro momento, o campo institucional codifica princípios institucionais em regras e rotinas e, na sequência, os atores, através de suas ações e interações - campo da ação - incorporam as regras e rotinas que codificam os princípios institucionais.

O comportamento repetitivo dos atores provoca a repetição de regras e rotinas e finalmente essas rotinas e regras se tornam institucionalizadas, constituindo novos elementos do campo institucional. 


\section{Primeiro Processo: Codificação (seta a)}

Este processo requer que os princípios institucionais idealizados sejam codificados, isto é, detalhados e especificados em rotinas e regras. 0 processo de codificação é orientado pela arquitetura do conjunto de princípios desejados, mas também é impactado pelas rotinas e regras existentes. No processo de concepção ou modelagem de um novo sistema de contabilidade gerencial é necessário entender as premissas de gestão e os modelos decisórios dos gestores, ou seja, identificar o campo institucional da organização. A partir daí o processo de modelagem do novo sistema de contabilidade gerencial se completa, com a definição de premissas, a especificação de conceitos, e a definição de critérios analíticos de mensuração e de identificação de resultados com objetos de gestão. Fundamentalmente o processo de concepção de um sistema de contabilidade gerencial corresponde à etapa de codificação caracterizada no modelo da figura 1 .

\section{Segundo Processo: Incorporação (seta b)}

Envolve os atores incorporando as rotinas e regras, as quais codificam os princípios institucionais desejados pela organização. 0 processo de incorporação pode envolver escolha consciente, mas normalmente é resultado de um monitoramento reflexivo e da aplicação do conhecimento tácito a respeito de como as coisas devem ser feitas. A incorporação de novas rotinas e regras pode ficar sujeita a resistências, especialmente se as novas rotinas e regras desafiarem os significados e valores existentes e se os atores tiverem suficiente poder para interferir no processo.

\section{Terceiro Processo: Reprodução (seta c)}

Ocorre à medida que o comportamento repetitivo conduz à reprodução de rotinas. Burns e Scapens (2000) observam que esta reprodução pode envolver escolha consciente ou inconsciente. A ocorrência da escolha consciente é mais provável quando os atores são capazes de reunir elementos que permitam coletivamente questionar as rotinas e regras existentes. Soin, Seal e Cullen (2002, p. 255) observam que "[...] uma questão chave na avaliação de mudanças diz respeito à questão da reprodução. As mudanças foram realmente incorporadas em novas rotinas e regras ou elas são simplesmente intervenções passageiras?"

\section{Quarto Processo: Institucionalização (seta d)}

A institucionalização de regras e de rotinas reproduzidas por meio de comportamentos repetitivos dos atores individuais pressupõe que os padrões de comportamento atuais sejam desassociados de suas circunstâncias históricas.

A pesquisa apresentada a seguir é centrada no primeiro processo da estrutura conceitual apresentada: a Codificação. 


\section{ESTUDO DE CASO}

\subsection{OBJETIVO E METODOLOGIA}

O objetivo deste estudo de caso (YIN, 2001) foi avaliar o processo de implementação do sistema de custos em uma organização bancária denominada, neste estudo, de Banco Sigma. A investigação foi focada especificamente na modelagem do sistema que, sob o prisma da estrutura conceitual de Burns e Scapens (2000), corresponde à fase da codificação das regras e rotinas a partir dos princípios do campo institucional da organização. 0 desenvolvimento da pesquisa ocorreu na fase em que a instituição estava validando o modelo conceitual de seu novo sistema de custos e iniciando o planejamento da sua implantação.

Os dados foram obtidos por meio de entrevistas e de análise de documentos. As entrevistas foram conduzidas junto ao gestor do processo de mudança, executivo da área de Controladoria, responsável pela implementação do sistema. Foram realizadas cinco entrevistas de aproximadamente duas horas no período de aproximadamente três meses com o objetivo de conhecer (i) os antecedentes ao processo de mudança, (ii) as características organizacionais e operacionais do banco e (iii) o processo de modelagem utilizado para a concepção do sistema.

Com o objetivo de conferir um maior grau de confiabilidade às informações fornecidas pelo entrevistado, realizou-se um processo de triangulação, por meio de análise documental, pesquisando-se: (i) documentos oficiais da instituição, relativos à estrutura de organização, funcionamento de processos e atividades e relatórios financeiros, (ii) relatório de depoimentos dos executivos das diversas áreas do banco sobre necessidades, expectativas e percepção quanto à implantação do novo sistema, (iii) relatório da modelagem do sistema de custos estudado.

\subsection{CARACTERÍSTICAS DO NEGÓCIO}

A entidade estudada é um banco múltiplo inserido em um importante conglomerado financeiro privado que atua em território brasileiro. Este banco ocupa posição dentre as cinco maiores instituições financeiras classificadas pelo critério de valor total dos ativos no ano de 2005. Os principais produtos do banco são:

- Câmbio

- Cartões

- Seguros

- Cobrança

- Capital de Giro

- Conta Garantida 
- Depósitos à Vista

- Conta Remunerada

- Desconto de Títulos

- Crédito Direto ao Consumidor.

O banco é estruturado em três grandes níveis: (i) unidades de negócio, (ii) unidades de apoio e (iii) unidades responsáveis pela produção de serviços. As unidades de negócio dizem respeito às agências e pontos de venda e seus custos diretos representam $72 \%$ dos custos totais do banco.

As unidades de apoio são responsáveis por diversas atividades, sendo as mais representativas: Engenharia e Patrimônio, Marketing, Recursos Humanos, Jurídico, Controladoria, Contabilidade e Auditoria Interna, responsáveis por $8 \%$ dos custos da organização. As unidades de produção dos serviços correspondem à "fábrica" do banco, sendo Tecnologia de Informação a de destaque, embora diversas outras unidades de back office façam parte dessa classificação. 0 custo direto desses órgãos de produção corresponde a $20 \%$ do total dos custos do banco.

\subsection{ANTECEDENTES AO PROCESSO DE MUDANÇA}

Um tema comum na pesquisa de contabilidade gerencial, sob o prisma da teoria da contingência, é que mudanças no ambiente organizacional provocam mudanças nos sistemas de contabilidade gerencial (BAINES; LANGFIELD-SMITH, 2003). Nesta seção é realizada uma retrospectiva das circunstâncias que levaram à revisão do modelo conceitual do sistema de custos do banco objeto do estudo de caso.

O Banco Sigma investiu vultosos recursos materiais e humanos próprios por longo período de tempo e incorreu em gastos relevantes com empresa multinacional de consultoria para conceber e implantar um modelo conceitual de sistema de custos, que tinha por objetivo satisfazer a duas necessidades: (i) apuração de custos e resultados e (ii) gestão de custos.

0 modelo adotou um método híbrido, baseando-se na filosofia do Custeio por Absorção, do Custeio Variável e do Custeio por atividades (ABC), alocando os custos por contas, por centros de custos e por atividades até o nível de agência e de executivo de contas. Aos produtos eram apropriados os custos variáveis (todos diretos) e alocados apenas os indiretos relativos ao processamento de documentos e transações (Tecnologia de Informação). Os custos de overhead, representados pela unidade de negócio Corporação, aí incluídos o Conselho de Administração e a Diretoria Executiva, não eram repassados aos produtos nem aos clientes. Nesse sentido, Major e Hopper (2005) observam que a definição de $A B C$ não é clara e as empresas podem definir sua terminologia e métodos de diferentes formas e assim calcular diferentes custos baseados em atividades diferentemente e usá-los para diferentes propósitos.

O sistema contemplava cerca de sete mil centros de custos e aproximadamente 
duzentas e cinquenta atividades, sendo os dados processados no aplicativo OROS integrado ao sistema SAP. A percepção dos membros da alta administração era de que o sistema apresentava, pelo menos, dois grandes problemas: (i) falhas conceituais e (ii) excessivo tempo de processamento. Como consequência, o sistema não foi efetivamente institucionalizado na organização e encontrou forte resistência junto aos executivos do banco. Segundo depoimento adicional de um dos diretores do banco, "o sistema era extremamente complexo, que demandava excessivo tempo de processamento dos dados e as informações geradas não eram adequadamente compreendidas pelos gestores". Cooper e Slagmulder (1999) advertem para o cuidado que se deve ter contra os pressupostos minimalistas na modelagem do que eles denominam de sistemas de custos inteligentes.

Algumas das principais questões que incomodavam os gestores eram as seguintes:

- Quais são os verdadeiros custos variáveis dos produtos?

- Quais são os verdadeiros custos variáveis dos clientes?

- Além dos custos variáveis, que são diretos, que outros custos deveriam ser considerados no cômputo do custo unitário dos produtos?

- Além dos custos variáveis, que outros custos deveriam ser considerados no cômputo do custo por cliente?

- Além dos custos diretos (variáveis ou fixos), que outros custos deveriam ser considerados no cômputo do custo das unidades de negócio (diretorias, agências etc.)?

- Deveria ser apurado o custo unitário de cada produto?

- Em caso positivo, como definir o nível de capacidade para calcular os custos unitários?

- Que tratamento dar aos custos da capacidade não utilizada?

- Qual é o impacto das decisões dos gestores sobre os custos da sua unidade?

- Qual é o impacto da variação de volume sobre os custos do banco?

- Qual é o impacto da variação de eficiência sobre os custos do banco?

A diretoria do banco desejava ser mais rigorosa na cobrança de ações dos gestores das áreas no que se refere à gestão de custos e resultados, mas não dispunha de um sistema de informação que the desse suporte para isso. Disso decorreu a necessidade de se repensar o modelo conceitual do sistema de custos do banco. No primeiro semestre do ano de 2005, aproveitando a oportunidade de uma mudança na estrutura organizacional e considerando que o sistema de custos estava inoperante, a empresa resolveu repensar o modelo conceitual de seu sistema de custos e a área de Controladoria assumiu a responsabilidade pela condução desse projeto. 


\subsection{A IDENTIFICAÇÃO DO CAMPO INSTITUCIONAL}

O campo institucional representa uma estrutura de regras e tipificações existentes, derivadas de uma história acumulada de ações e interações. 0 enfoque institucional enfatiza influências culturais no processo de tomada de decisões e nas estruturas formais. Como Barley e Tolbert (1997) explicam, a teoria institucional enfoca as organizações e os indivíduos que as povoam, suspensos em uma rede de valores, normas, regras, crenças e pressuposições aceitas sem questionamentos. Esses elementos culturais definem o modo como o mundo é e como deveria ser; eles estabelecem um esquema para o detalhamento e especificação de formas de procedimentos que deveriam ser seguidos pelos membros de uma organização. Nesse sentido, as instituições representam restrições no processo de escolhas que os indivíduos e os grupos gostariam de fazer, embora essas restrições estejam o tempo todo abertas para modificações.

Do ponto de vista prático, Soin, Seal e Cullen (2002, p. 255) observam que “[...] a teoria sugere que a primeira etapa na aplicação da teoria é uma análise do campo institucional com a identificação do conjunto inicial de rotinas e regras que caracterizam o controle gerencial no banco." De fato as rotinas e regras materializadas em artefatos são expressões mais concretas do campo institucional, mas nesse campo também estão presentes, de forma subliminar, os valores e pressuposições aceitos pelo grupo social.

Conforme informações obtidas nas entrevistas, o processo de revisão do modelo conceitual do sistema de custeio do banco foi desenvolvido pela equipe do projeto em três etapas. Na primeira, foi realizada pesquisa interna com trinta e um executivos do banco para identificar as principais necessidades informativas desses gestores, bem como sua visão sobre os objetivos e as dimensões relevantes que deveria possuir o sistema de custos. Foram consultados membros do comitê executivo do banco, gestores das áreas comerciais, da área de processamento de transações bancárias, denominadas “fábricas”, e gestores das áreas de apoio. Para a coleta de dados foram aplicados questionários com questões abertas. Na segunda etapa, realizou-se um benchmarking com outras instituições financeiras de porte e características similares às do banco, procurando conhecer os modelos de custeio por elas utilizados, o tempo médio de implantação, os fatores críticos de sucesso e as armadilhas a serem evitadas. Na terceira, o modelo foi discutido com uma equipe de consultores externos.

Com base no aprendizado obtido com as três etapas descritas acima, a equipe do projeto, liderada pela área de Controladoria, iniciou o delineamento das premissas, dos princípios e das diretrizes que deveriam nortear a construção do novo modelo conceitual do sistema de custeio.

Verificou-se inicialmente que, embora a pesquisa interna revelasse que o full allocation era bem aceito pelos gestores da organização, não havia consenso quanto à abrangência do processo de alocação de custos aos produtos, ou seja, se deveria ser utilizada uma abordagem mais próxima ao custeio por absorção ou mais próxima ao custeio pleno. Havia consenso, no entanto, de que, independentemente 
da decisão sobre o grau de alocação de custos indiretos aos produtos, a apuração das variações de custos deveria ser realizada no nível do gestor que tivesse autonomia para implementar medidas corretivas.

A pesquisa interna revelou ainda que a principal preocupação dos executivos relacionava-se com a previsibilidade dos custos; assim, o sistema deveria ter a característica de proporcionar uma antevisão dos valores atribuídos, evitandose surpresas. Essa preocupação foi quase unânime entre os pesquisados, os quais deixaram claro que a previsibilidade dos valores dos custos, na sua visão, era mais importante do que a exatidão dos números apurados e do que a acurácia do sistema de custeio.

Outra importante constatação, convergente entre os pesquisados, foi quanto à utilidade de apurar-se o custo unitário dos produtos e de se avaliar o desempenho dos gestores das áreas processadoras de produtos também em função dos custos unitários. A partir daí surgiu o questionamento de que conceito de capacidade utilizar (teórica, prática, normal ou orçada) como base à apuração dos custos unitários e ao processo de avaliação de desempenho dos gestores.

Considerando as duas constatações: (i) a relevância do conhecimento do custo unitário dos produtos e (ii) a necessidade de sua previsibilidade, a equipe do projeto decidiu-se pelo desenvolvimento de um sistema de custo padrão e decidiu que as áreas usuárias dos produtos (agências) deveriam ter conhecimento prévio do custo padrão unitário dos produtos. É importante observar que esses aspectos se tornam mais desafiadores em ambiente operacional de alto volume de custo fixo e de volumes de produção oscilantes.

Com base na pesquisa realizada foram definidos os seguintes princípios que deveriam nortear todo o processo de concepção do novo modelo de custeio:

a) O sistema deveria prover efetivo suporte ao processo de gestão e ser consistente com o novo modelo de estrutura organizacional.

b) O sistema deveria refletir a natureza dos processos do negócio.

c) O sistema deveria possibilitar total transparência do processo de formação de custos.

d) As informações geradas pelo sistema de custeio deveriam dar suporte ao processo de racionalização de custos na organização.

e) As informações geradas pelo sistema de custeio deveriam dar suporte ao processo de avaliação de desempenho na organização.

f) O sistema deveria contemplar três grandes entidades objeto de custeio: produtos, clientes e unidades de negócio.

g) Não deveria haver arbitrariedades no processo de alocação de custos.

h) O sistema de custeio deveria dar segurança aos gestores quanto à previsibilidade dos valores mensurados. 


\subsection{O PROCESSO DE CODIFICAÇÃO}

Do ponto de vista de desenvolvimento de sistemas, o processo de codificação corresponde à fase de modelagem do sistema, ou seja, à especificação do modelo conceitual e lógico que deverá orientar as soluções de tecnologia de informação. A abordagem normativa em contabilidade gerencial menciona que o modelo deve ser especificado de forma a gerar informações eficazes para o processo de tomada de decisões gerenciais racionais (BJORNENAK; OLSON, 1999). Nesse contexto, Kaplan (1990) relata que as empresas estão desenvolvendo novos sistemas de custos para produzir informações úteis para o controle operacional e para a análise da rentabilidade de produtos, linhas de produtos e clientes. Brignall (1997), bem como Auzair e Langfield-Smith (2005), propõem que o desenho do sistema de contabilidade gerencial deve ser efetuado com a consciência dos fatores contingentes relevantes, considerando a missão e estratégia da organização e considerando também o tipo de processo do serviço. Abernethy et al. (2001) desenvolveram estudo para examinar as implicações da diversidade de produtos nas escolhas de modelagem de sistemas de custos.

À luz da abordagem institucional, o processo de codificação requer que os princípios institucionais sejam codificados, isto é, detalhados e especificados em rotinas e regras. Burns e Scapens (2000) observam que o processo de codificação é influenciado (i) pelas rotinas e instituições vigentes, (ii) pelas intenções (racionais) dos agentes de mudanças e (iii) por outras instituições fora da organização. As novas regras e rotinas codificadas são influenciadas pelas regras e rotinas vigentes e nesse sentido elas terão mais chance de ser implementadas à medida que forem mais consistentes com as regras e rotinas amplamente aceitas no âmbito da organização. Por outro lado, o processo de codificação também é fortemente influenciado pela intenção consciente e racional dos agentes de mudança. No bojo dessas intenções destaca-se aquilo que se deseja eliminar, ou seja, os conteúdos das rotinas e regras que não se conformam com os valores do grupo social, e também aquilo que se deseja adicionar, ou seja, os elementos que reflitam da melhor forma possível preocupações com eficiência econômica, controle de custos e tomada de decisões gerenciais. Os agentes de mudança também serão influenciados por outras instituições normalmente fora da organização, tais como práticas utilizadas em empresas pesquisadas (melhores práticas), opiniões de consultores, recomendações de associações profissionais e ainda por grupos que exerçam algum tipo de poder sobre o time do projeto.

Com base nos princípios e diretrizes estabelecidos em função (i) das informações obtidas com a pesquisa interna realizada junto aos executivos, (ii) do benchmarking realizado e (iii) das reuniões com os consultores externos, a equipe da Controladoria, condutora do projeto, definiu os conceitos de mensuração a serem utilizados no novo modelo do sistema de custeio. Do ponto de vista do modelo de Burns e Scapens (2000), esta etapa corresponde ao processo de codificação, em que os princípios institucionais são codificados em regras e rotinas. No estudo em questão pode-se observar a especificação dos seguintes conceitos: 


\section{(i) Separação entre o custo de produto e o custo de estrutura}

Deveria haver separação entre o custo do produto e o custo da estrutura. Os produtos deveriam absorver todos os custos de produção, sendo estes caracterizados como os custos pertencendo às unidades (até o nível de gerência) responsáveis pelos processos produtivos.

Os custos nos níveis de Superintendência e Diretoria não seriam alocados aos produtos. Essa definição atendia aos anseios dos gestores quanto à adoção do full allocation e resolvia a questão do limite de nível organizacional até o qual os custos deveriam ser alocados aos produtos. Assim, seriam alocados aos produtos todos os custos das áreas processadoras, denominadas "fábricas".

O trabalho de Brignall et al. (1991) reporta que somente duas empresas de serviços dentre cinco pesquisadas possuíam sistema de custos baseado no custeio pleno (full costing). Todas as demais empresas de serviços estavam competindo com base em diferenciação, e planejavam e controlavam seus custos no nível de centro de custo funcional e não no nível do produto.

\section{(ii) Custo padrão dos produtos}

Deveria haver custo padrão para os produtos do banco. Esta definição atendia aos anseios dos gestores no que se refere à segurança quanto à previsibilidade dos valores no exercício das suas atividades. Assim, o sistema seria alimentado com valores projetados (orçados) tanto nas suas variáveis monetárias (custos) como físicas (volume de transações).

Para formulação dos padrões, deveria ser utilizada a capacidade prática e o sistema de padrões deveria ser integrado ao sistema orçamentário. Os valores dos custos reais deveriam ser consistentes com os da contabilidade. 0 gerente do projeto mencionou que "um ponto relevante do novo modelo era o fato de que o custo unitário dos produtos não seria impactado pelo volume real de transações do período em curso e, por utilizar o custo-padrão, os gestores não teriam surpresas quanto ao custo unitário que thes seria debitado."

\section{(iii) Apuração de resultados por produtos, clientes e unidades de negócio}

Deveria ser apurada a margem de contribuição dos produtos e dos clientes considerando-se as receitas líquidas e os custos diretos dos produtos. Nesse sentido, o líder da equipe condutora do projeto no banco afirmou: "Um dos principais benefícios do novo modelo foi a compreensão de que existem diferentes margens a serem analisadas e que cada uma delas tem um significado diferente."

As unidades de negócio (agências, executivos de contas etc.) deveriam ser debitadas pelo valor de custo dos produtos por elas comercializados, tomando-se por base os custos padrão e os volumes reais de cada período. Além disso, essas unidades 
de negócio deveriam absorver os custos de overhead relativos à administração central diretamente, isto é, sem a passagem destes pelo custo dos produtos.

Os custos das áreas de apoio da administração central deveriam ser debitados, num primeiro estágio, às quatro diretorias - Atacado, Varejo, Private e Coligadas - proporcionalmente à Margem de Contribuição Meta, conforme estabelecido no orçamento. No segundo estágio, o custo de cada diretoria deveria ser alocado às suas respectivas unidades também proporcionalmente aos valores-meta estabelecidos em orçamento. Como exemplo, esse seria o critério para alocar o custo da Diretoria de Varejo às agências. O valor do overhead assim alocado às unidades de negócio deveria ser suportado por elas como uma espécie de pagamento de franquia pelo uso da marca do banco e dos serviços recebidos das áreas de administração central, tais como marketing, jurídico e outras.

\section{(iv) Custeio baseado em atividades}

Deveria ser utilizada a base conceitual do Custeio Baseado em Atividades (ABC) para custear os produtos. A literatura sobre o sistema $A B C$ privilegia sua utilidade quando aplicado a todo o overhead da organização e, no caso do Banco Sigma, foi decidida sua implementação somente no ambiente das "fábricas" de produtos. Nesse contexto, Drury e Tayles (1998) observam que não existe um sistema de custos ideal e que as escolhas dependem das circunstâncias.

A decisão tomada pela equipe do projeto sobre a forma de aplicação do sistema $\mathrm{ABC}$ atendeu à diretriz estabelecida de não se cometer arbitrariedades no processo de alocação de custos, uma vez que os direcionadores de custos devem refletir verdadeiras relações de causa e efeito. Foi observado que, no desenvolvimento dos trabalhos de concepção do modelo conceitual do sistema, obedeceu-se a tipologia clássica do sistema de custeio baseado em atividades nos seus três módulos: (i) recursos, (ii) atividades e (iii) objetos de custeio.

Quanto ao primeiro módulo, o dos recursos, estes foram inicialmente classificados em custos variáveis e custos fixos, com o objetivo de gerar condições para o cálculo da margem de contribuição de produtos e clientes de acordo com a metodologia de custeio variável. Nesse contexto, foram identificados os principais custos variáveis de produtos do banco: (i) telemarketing, (ii) tarifa de cartórios, (iii) tarifa de compensação, (iv) tarifa interbancária (Tib) e (v) material, denominado custo tangível. Os principais custos fixos identificados foram (i) aluguel, (ii) depreciação, (iii) salários e encargos sociais. No que diz respeito ao segundo módulo, foram identificadas as seguintes atividades:

- Atender clientes VIP.

- Processar operações.

- Aprovar e conceder crédito.

- Assessorar o auto-atendimento. 
- Prover suporte em controladoria.

- Prover suporte em assuntos jurídicos.

- Gerenciar o relacionamento com clientes.

- Prover suporte em assuntos de marketing.

- Prover suporte em tecnologia de informação.

No que diz respeito ao processo de identificação dos direcionadores de custos de primeiro estágio (direcionadores de custos dos recursos), a equipe do projeto decidiu pela não adoção do sistema de apontamentos através de time sheet para custear as atividades, entendendo que, pela experiência do banco, essa prática, além de contrariar a sua cultura organizacional, seria contraproducente, pois absorveria precioso tempo das pessoas e a relação entre o custo e o benefício dessa prática seria desfavorável. Decidiu-se que as atividades seriam custeadas com base em entrevistas e em levantamento de tempos realizados periodicamente.

Quanto aos direcionadores de custos de segundo estágio (direcionadores de custos das atividades), para alocar aos produtos os custos do processamento de operações, foram observados os respectivos processos e atividades e entrevistados os gestores responsáveis. Como resultados, foram identificados os seguintes direcionadores: (i) tempo de máquina, (ii) tempo das pessoas e (iii) volume de transações. Quanto ao terceiro módulo, referente às entidades objeto de custeio, foram definidas as seguintes entidades:

- Produtos: Câmbio, Poupança, Cobrança, Conta Corrente, Empréstimos etc.

- Unidades de Negócio: atacado, varejo, private bank e agências.

- Clientes: clientes individuais, classes ou categorias de clientes.

\section{DISCUSSÃO DO CASO}

Conforme mencionado anteriormente, a modelagem, também denominada concepção do modelo conceitual de um sistema de custos, equivale ao primeiro processo do modelo de Burns e Scapens (2000) denominado codificação. O processo de codificação se desenvolve inicialmente a partir da identificação dos princípios institucionais idealizados. Soin, Seal e Cullen (2002) observam que o primeiro passo na aplicação do modelo de Burns e Scapens (2000) é a análise do campo institucional vigente (institutional realm). Após essa fase, desenvolve-se a codificação propriamente dita, ou seja, os princípios institucionais idealizados são codificados, isto é, detalhados e especificados em rotinas e regras.

A identificação dos princípios institucionais é fundamental para o sucesso da concepção do sistema e deve ser efetuada de forma consciente pela equipe do projeto, uma vez que esses princípios idealizados serão especificados em conceitos; em última 
instância, serão materializados em software em tecnologia de informação.

No caso em estudo, pode-se perceber que o sistema antecedente não foi institucionalizado não apenas por motivos técnicos (tempo de processamento e baixa compreensão dos usuários), mas fundamentalmente porque não atendia às expectativas dos usuários. O software comprado pelo banco e implantado pelos consultores externos não refletia os princípios institucionais da organização. Esse fracasso, provavelmente, fez com que a equipe do projeto ficasse mais atenta, no sentido de captar os desejos, percepções e necessidades dos gestores do banco. Conforme pode ser analisado no caso Omega relatado em Burns e Scapens (2000), um novo sistema de contabilidade gerencial (rotinas e regras codificados) que apresente contradições em relação às rotinas e regras existentes (campo institucional vigente) pode trazer conflito e resistência, levando ao insucesso na implementação do novo sistema.

As informações coletadas neste estudo demonstram que, no desenvolvimento do novo sistema, a equipe do projeto estava muito consciente no sentido de captar as opiniões dos executivos do banco, desenvolvendo pesquisa com mais de trinta e um executivos da instituição. Os dados do estudo demonstram que a equipe do projeto identificou os princípios institucionais idealizados pelos executivos do banco relativos a sistema de custos.

O processo de codificação (especificação de conceitos), conforme se pode observar pelas informações da seção 3.5 deste trabalho, foi desenvolvido pela equipe do projeto de acordo com a orientação de atender efetivamente as visões dos executivos da empresa. Nesse sentido, esse processo de codificação, que envolveu a especificação de conceitos e o detalhamento de regras e rotinas, se deu de forma coerente com os valores do campo institucional da organização bancária. Um aspecto de destaque nesse processo é que não ocorreu uma submissão cega às opiniões dos gestores. A preocupação com o aspecto institucional caminhou em paralelo com a dimensão racional-normativa, no sentido da mensuração de eficiências e de rentabilidade de produtos e unidades de negócios. Nesse sentido, a equipe do projeto realizou benchmarking com outras instituições financeiras de porte e características similares às do banco referente a modelos de custeio utilizados e experiências de implementação de sistemas, bem como discutiu o modelo idealizado com uma equipe de consultores especialistas.

\section{CONSIDERAÇÕES FINAIS}

O objetivo desta pesquisa foi estudar o processo de modelagem do sistema de custos do Banco Sigma, descrevendo "como" ocorreu o processo de codificação das regras, rotinas e conceitos do sistema a partir da identificação dos princípios institucionais da organização. O arcabouço conceitual utilizado no estudo foi o modelo de Burns e Scapens (2000).

A primeira conclusão observada no estudo, em linha com as diretrizes do 
arcabouço teórico, é que a dimensão institucional é tão importante quanto a dimensão técnico-racional, na fase de concepção do modelo conceitual do sistema. Isso significa dizer que não basta planejar racionalmente os melhores conceitos, rotinas e regras para um novo sistema, mas também que essa base conceitual planejada esteja em sintonia com as diretrizes do campo institucional da organização. Essa sintonia somente será possível através da identificação das crenças e valores que permeia a organização e que faz parte do denominado campo institucional.

No caso do Banco Sigma, pode-se perceber que houve um processo consciente de identificação de princípios institucionais e que o processo de codificação do novo sistema especificou conceitos e rotinas coerentes com os princípios institucionais compartilhados pelos executivos do banco.

Considerando-se as premissas da teoria institucional, é possível afirmar que, independentemente dos aspectos conceituais técnicos, o sistema modelado tem grande probabilidade de ser institucionalizado, ou seja, de se converter em um instrumento efetivamente aceito na instituição. Essa probabilidade de sucesso de institucionalização está diretamente correlacionada com a lógica de especificação dos conceitos do novo sistema. A lógica institucional indica que a fase de codificação das novas regras e rotinas deve ser orientada pelas crenças e valores presentes no campo institucional vigente na empresa.

No caso do Banco Sigma, pode-se perceber que houve um processo consciente de identificação de princípios institucionais e que no processo de codificação foram definidos e detalhados conceitos e rotinas coerentes com os princípios institucionais do banco.

Apesar das limitações metodológicas, o estudo traz contribuições efetivas, das quais a principal delas é proporcionar uma estrutura conceitual para a modelagem de sistemas de custos que propicie uma maior probabilidade de sucesso durante o processo de sua implantação. O estudo adiciona novos insights na construção do conhecimento da contabilidade de custos, dando ênfase à dimensão institucional relativamente à dimensão racional-normativa.

Finalmente o estudo contribui para a compreensão mais adequada dos fenômenos reais de mudança e institucionalização de sistemas de custos a partir da estrutura conceitual proposta por Burns e Scapens (2000). Neste contexto fica evidenciado que a visão normativa é necessária, no sentido da adoção de melhores práticas de gestão, porém, a visão institucional é igualmente importante, no sentido de se assegurar a efetiva institucionalização do sistema idealizado.

\section{REFERÊNCIAS}

ABERNETHY, M. A.; LILLIS, A. M.; BROWNELL, P.; CARTER, P. Product diversity and costing system design choice: field study evidence, Management Accounting Research, 12, p. 261-279, 2001. 
AUZAIR, S. M.; LANGFIELD-SMITH, K. The effect of service process type, business strategy and life cycle stage on bureaucratic MCS in service organizations. Management Accounting Research, 16, p. 399-421, 2005.

BAINES, A.; LANGFIELD-SMITH, K. Antecedents to management accounting change: a structural equation approach. Accounting, Organizations and Society, 28, p. 675698, 2003.

BARLEY, S. R.; TOLBERT, P. S. Institutionalization and structuration: studying the links between action and institution. Organization Studies, 18 (1), p. 93-117, 1997.

BJORNENAK, T.; OLSON, O. Unbundling management accounting innovations. Management Accounting Research, 10, p. 325-338, 1999.

BRIGNALL, S. A contingent rationale for cost system design in services. Management Accounting Research, 8, p. 325-346, 1997.

BRIGNALL, T. J.; FITZGERALD, L.; JOHNSTON, R.; SILVESTRO, R. Product costing in service organizations. Management Accounting Research, 2, p. 227-248, 1991.

BRIGTH, J.; DAVIES, R. E.; DOWNES, C. A.; SWEETING, R. C. The deployment of costing techniques and practices: a UK study. Management Accounting Research, v. 3, n. 3, p. 201-212, 1992.

BURNS, J. The dynamics of accounting change: inter-play between new practices, routines, institutions, power and politics. Accounting, Auditing \& Accountability Journal, v. 13, n. 5, p. 566-596, 2000.

BURNS, J. The institutionalization of accounting routines: Keano Ltd. Proceedings of the 1995 Management Control Association Symposium, p. 217-233, 1997.

BURNS, J.; SCAPENS, R. W. Conceptualizing management accounting change: an institutional framework. Management Accounting Research, v. 11, p. 3-25, 2000.

COBB, I.; HELLIAR, C.; INNES, J. Management accounting change in a bank. Management Accounting Research, 6, 155-175, 1995.

COHEN, J. R.; PAQUETTE, L. Management accounting practices: perceptions of controllers. Journal of Cost Management for the Manufacturing Industry, p. 73-83, 1991.

COOPER, R.; SLAGMULDER, R. Intelligent cost system design. Strategic Finance, 80, p. 18, 1999.

COVALESKI, M. A.; DIRSMITH, M. W.; SAMUEL, S. Managerial accounting research: the contributions of organizational and sociological theories. Journal of Management Accounting Research, 8, p. 1-35, 1996.

DRURY, C.; TAYLES, M. Cost system design for enhancing profitability. Management Accounting, 76, p. 40, 1998. 
DRURY, C.; TAYLES, M. Issues arising from surveys of management accounting practices. Management Accounting Research, v. 6, p. 267-280, 1995.

EDWARDS, K. A.; EMMANUEL, C. R. Diverging views on the boundaries of management accounting. Management Accounting Research, v. 1, n. 1, p. 551-563, 1990.

EMORE, J. R.; NESS, J. A. The slow pace of meaningful changes in cost systems. Journal of Cost Management for the Manufacturing Industry, Winter, p. 36-45, 1991.

GIDDENS, A. The Constitution of Society. Cambridge: Polity Press, 1984.

GRANLUND, M. Towards explaining stability in and around management accounting systems. Management Accounting Research, v. 12, p. 141-146, 2001.

GRANLUND, M.; LUKKA, K. It's a small world of management accounting practices. Journal of Management Accounting Research, 10, p. 153-179, 1998.

GREEN, F. B.; AMENKHIENAN, F. E. Accounting innovations: a cross sectional survey of manufacturing firms. Journal of Cost Management for the Manufacturing Industry, Spring, 1992.

GUERREIRO, R.; CASADO, T.; BIO, S. R. Some reflections on the archetypes in cost accounting: an exploratory study. Journal of Applied Management Accounting Research, 2(1), p. 41-54, 2004.

GUERREIRO, R.; PEREIRA, C. A.; FREZATTI, F. Evaluating management accounting change according to the institutional theory approach: a case study of a Brazilian bank. Journal of Accounting \& Organizational Change, 2, p. 196-228, 2006.

HELLIAR, C.; COBB, I.; INNES, J. A longitudinal case study of profitability reporting in a bank. British Accounting Review, 34, p. 27-53, 2002.

JOHNSON, H. T.; KAPLAN, R. S. Relevance lost-the rise and fall of management accounting. Boston: Harvard Business School Press, 1987.

KAPLAN, R. The four-stage model of cost systems design. Management Accounting, 71, 8, p. 22, 1990.

KASURINEN, T. Exploring management accounting change: the case of balanced scorecard implementation. Management Accounting Research, v.13, p. 323-341, 2002.

LIBBY, T.; WATERHOUSE, J. H. Predicting change in management accounting system. Journal of Management Accounting Research, 8, 137-150, 1996.

LUKKA, K. Management accounting change and stability: Loosely coupled rules and routines in action, Management Accounting Research, (article in press - doi: 10.1016/j.mar.2006.06.006), 2006.

MAJOR, M.; HOPPER, T. Managers divided: implementing $A B C$ in a Portuguese telecommunications company. Management Accounting Research, 16, p. 205-229, 
2005.

OLIVER, C. Sustainable competitive advantage: combining institutional and resourcebased views. Strategic Management Journal. v. 18, n. 9, p. 697-713, 1997.

QUATTRONE, P.; HOPPER, T. What does organizational change mean? Speculations on a taken for granted category. Management Accounting Research,12, p. 403-435, 2001.

SCAPENS, R. W. Never mind the gap: towards an institutional perspective on management accounting practice. Management Accounting Research, 5, p. 301-321, 1994.

SCAPENS, R. W. Understanding management accounting practices: a personal journey. The British Accounting Review, 38, p. 1-30, 2006.

SCAPENS, R. W.; ROBERTS, J. Accounting and control. A case study of resistance to accounting change. Management Accounting Research, 5, p. 301-321, 1993.

SITI-NABIHA, A. K.; SCAPENS R. W. Stability and change: an institutional study of management accounting change. Accounting, Auditing \& Accountability Journal, 18, p. 44-73, 2005.

SOIN, K.; SEAL, W.; CULLEN, J. ABC and organizational change: an institutional perspective. Management Accounting Research, 13, p. 249-271, 2002.

SPRAAKMAN, G. The impact of institutions on management accounting changes at the Hudson's Bay Company, 1670 to 2005. Journal of Accounting \& Organizational Change, 2, p. 101-122, 2006.

YIN, R. Estudo de caso: planejamento e métodos. 2. ed. Porto Alegre: Ed. Bookman, 2001. 


\section{ENDEREÇO DOS AUTORES:}

\section{Welington Rocha}

Universidade de São Paulo

Faculdade de Economia Administração e Contabilidade

Av. Luciano Gualberto, 908 - FEA 3

Cidade Universitária

São Paulo, SP - Brasil

05508-900

\section{Reinaldo Guerreiro}

Universidade de São Paulo

Faculdade de Economia Administração e Contabilidade Av. Luciano Gualberto, 908 - FEA 3

Cidade Universitária

São Paulo, SP - Brasil

05508-900 